\title{
RESEARCH HIGHLIGHT ILC transdifferentiation: roles in cancer progression
}

\author{
Maryam Ghaedi ${ }^{1}$ and Pamela S. Ohashi ${ }^{1,2}$ \\ Cell Research (2020) 30:562-563; https://doi.org/10.1038/s41422-020-0326-5
}

\begin{abstract}
Innate lymphoid cells (ILCs) play key roles in determining the outcome of immune responses and their roles in shaping tumor immunity have begun to be deciphered. Wang et al. demonstrate that dynamic conversions of ILC subsets and functions might promote colorectal cancer (CRC) progression; hence, targeting ILCs may present a potential novel approach to immunotherapy.
\end{abstract}

Innate lymphoid cells (ILCs) include cytotoxic natural killer (NK) cells and cytokine-producing ILCs. ILCs lack antigen-specific receptors and primarily respond to cytokines. ${ }^{1}$ ILCs are divided into group 1 (ILC1s), group 2 (ILC2s), and group 3 (ILC3s), based on functional similarities to T helper cell subsets. ILC1s produce IFN- $\gamma$ and require the transcription factor T-bet for their development and function. ILC2s produce IL-5 and IL-13 and require GATA3 and RORa. ILC3s produce IL-22 and/or IL-17 and require RORyt. A distinct regulatory ILC population (ILCreg), which produces IL-10 and requires ID3 has also been described. ${ }^{2}$ ILCs express a variety of other effector molecules and influence the innate and adaptive immune responses. ${ }^{1}$ It is becoming more evident that within each ILC group, distinct subsets produce distinct effector molecules and mediate specific responses. ILC phenotype and function is closely imprinted by signals from the tissue microenvironment, ${ }^{3}$ thereby showing extensive heterogeneity across different tissues. ILCs have also been shown to display substantial plasticity in their phenotypes and effector functions.

ILCs have been found in human cancers ${ }^{4}$ and their roles in tumor immunity and progression remain to be fully clarified. ILC1s and NK cells may promote tumor immunosurveillance and clearance through IFN $-\gamma$ production. However, a population of $\mathrm{CD} 56^{+} \mathrm{CD}^{-}$cells has been shown to directly suppress anti-tumor $T$ cell responses from high-grade serous cancer (HGSC) patients. ${ }^{5}$ The presence of these cells was associated with a significant reduction in the recurrence-free survival of HGSC patients. ILC2s were reported to play important roles in the initiation and maintenance of adaptive Th2 and regulatory $T$ (Treg) cell responses, which might contribute to cancer progression. ILC2s promoted Th2 differentiation by their expression of IL-13. ${ }^{6}$ ILC2s elicited ICOS/ligand- and GITR/ligand-dependent Treg cell activation. ${ }^{7}$ In addition, IL-13 production by ILC2s promoted immunosuppression by the recruitment and activation of myeloid-derived suppressor cells in acute promyelocytic leukemia and non-muscleinvasive bladder cancer. ${ }^{8,9}$ Anti-tumor roles of ILC2s have also been described. IL-5 production by ILC2s promoted eosinophilia and reduced lung metastasis in melanoma-bearing mice. ${ }^{4}$ ILC3s have been shown to promote the formation of tertiary lymphoid structures in non-small cell lung cancer, which in turn may be related to protective immunity and better survival. ${ }^{10}$ However, high levels of IL-22, which is produced by ILC3s, have had implications in promoting gut tumorigenesis.
In a recent paper published in Cell Research, Wang and colleagues, using azoxymethane/dextran sodium sulfate (AOM/ DSS) induced colorectal (CRC), profiled tumor-infiltrating ILCs in the early and late stages of cancer progression by single-cell RNA sequencing (scRNA-seq), flow cytometry and functional assays. ${ }^{11}$ Collectively, six tumor-infiltrating ILC populations including ILC1, three ILC2 subsets (termed ILC2-A, B, C), ILC3 and ILCreg were identified. Wang et al. demonstrated that ILC1s underwent changes consistent with impairment of their immune function. In late stage CRC, ILC1s were decreased. They upregulated the expression of inhibitory receptors, downregulated IL-12RB2 and produced less IFN- $\gamma$. In addition, in advanced CRC patients, ILC1s were decreased in frequency and expressed higher levels of inhibitory receptors. Among the three ILC2 subsets, ILC2-A was the dominant subset in the early stage and was replaced by ILC2-C in the late stage. ILC2-Cs highly expressed heparan sulfate 3-O-sulfotransferase 1 (HS3ST1) and the co-inhibitory molecule programmed cell death protein 1 (PD-1). In advanced CRC patient samples, ILC2s also expressed HS3ST1 and PD-1. Transfer of PD $1^{\text {high }}$ ILC2s along with primary tumor cells from advanced CRC patients into immunocompromised NOD-Prkdc $c^{\mathrm{scid}} / \mathrm{L} 2 \mathrm{rg}^{\mathrm{tm} 1} / \mathrm{Bcgen}$ (B-NSG) mice resulted in increased tumor growth compared to the transfer of peri-tumor PD1 ${ }^{-}$ILC2s. ILC2s deficient in either HS3ST1 or PD-1 were unable to promote CRC progression. Furthermore, anti-PD-1 antibody treatment inhibited the impact of ILC2s on tumor growth. Moreover, ILC3s transdifferentiated into ILCregs, which produced IL-10 and enhanced tumor growth. TGF- $\beta$ facilitated this transdifferentiation, during which ILC3s downregulated RORyt expression and upregulated ID3 and IL-10. Hence, profiling ILCs at both early and late stages of CRC in this study uncovered their dynamic changes in proportion, gene expression profile and function, which were associated with tumor progression (Fig. 1). However, it should be noted that AOM/DSS induced CRC and the evaluation of the growth of patientderived xenografts in the immunocompromised B-NSG mouse models do not properly capture the contribution of adaptive immunity and in particular T cells. Hence, the overall impact of the aforementioned ILC subsets to CRC immunobiology remains unclear.

A recent study ${ }^{12}$ has shown that ILC2s promoted dendritic cell recruitment, $\mathrm{CDB}^{+} \mathrm{T}$ cell activation and rejection of immunogenic pancreatic ductal adenocarcinomas (PDACs) in mouse models. In this context, PD-1 was a negative regulator of ILC2 function and PD-1 blockade along with IL-33-induced activation of ILC2s maximally enhanced tumor control. In addition, ILC2s and activated $\mathrm{CD}^{+} \mathrm{T}$ cells were enriched in heavily infiltrated PDACs from long-term survivors compared to poorly inflamed tumors from short-term survivors. Tumor-infiltrating ILC2 frequency in

${ }^{1}$ Princess Margaret Cancer Centre, University Health Network, Toronto, ON, Canada and ${ }^{2}$ Department of Immunology, University of Toronto, Toronto, ON, Canada Correspondence: Pamela S. Ohashi (Pam.Ohashi@uhnresearch.ca)

Published online: 4 May 2020 


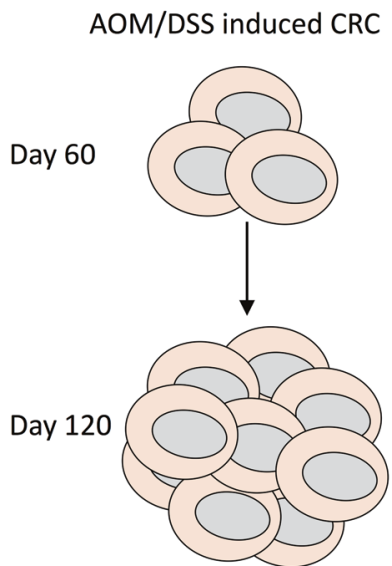

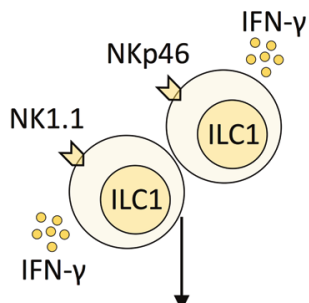

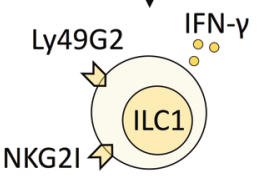

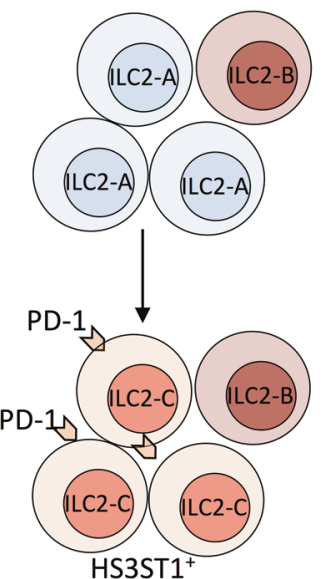

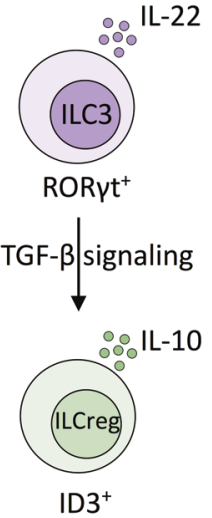

Fig. 1 ILCs dynamically change during CRC progression and contribute to cancer progression. ILCs were profiled in AOM/DSS induced colitis-associated CRC in early (day 60) and late (day 120) stages of cancer progression. ILC1s expressed activating receptors in the early CRC stage. These cells were reduced in numbers, upregulated the expression of inhibitory receptors and produced less IFN- $\gamma$ in the late stage. Two of the three distinct ILC2 subsets were present in early and late stages of CRC. ILC2-A was the dominant ILC2 subset in the early stage and was replaced by ILC2-C subset in the late stage. ILC2-Cs expressed HS3ST1 and PD-1. TGF- $\beta$ facilitated the transdifferentiation of IL-22-producing ILC3s in the early stage to IL-10-producing ILCregs in the late stage. These dynamic changes in ILCs contributed to tumor progression.

PDACs correlated with immune cytolytic activity and patient survival. Hence, ILC2s activated anti-tumor immunity and enhanced the effectiveness of anti-PD-1 immunotherapy in PDACs. Thus, ILC2s might limit or promote anti-tumor immune responses depending on the context.

The number of studies addressing the impact of ILCs in shaping tumor immunobiology and response to immunotherapy is increasing. However, many aspects of ILC biology should be evaluated. Primarily, the roles of ILCs in shaping myeloid-, DC- and $\mathrm{T}$ cell-mediated responses in tumor should be fully characterized in a tissue-specific manner. Moreover, the impact of ILC heterogeneity and plasticity over the course of tumor progression should also be considered. ILCs share many stimulatory and inhibitory pathways with $\mathrm{T}$ cells and current immunotherapeutic strategies can potentially target both ILCs and T cells. Hence, strategies to exploit ILC biology may be synergistic for promising immunotherapies.

\section{ADDITIONAL INFORMATION}

Competing interests: The authors declare no competing interests.

\section{REFERENCES}

1. Vivier, E. et al. Cell 174, 1054-1066 (2018).

2. Wang, S. et al. Cell 171, 201-216 (2017).

3. Ricardo-Gonzalez, R. R. et al. Nat. Immunol. 19, 1093-1099 (2018).

4. Ercolano, G., Falquet, M., Vanoni, G., Trabanelli, S. \& Jandus, C. Front. Immunol. 10, 1-9 (2019).

5. Crome, S. Q. et al. Nat. Med. 23, 368-375 (2017).

6. Halim, T. Y. F. et al. Immunity 40, 425-435 (2014).

7. Rauber, S. et al. Nat. Med. 23, 938-944 (2017).

8. Trabanelli, S. et al. Nat. Commun. 8, 1-14 (2017).

9. Chevalier, M. F. et al. J. Clin. Invest. 127, 2916-2929 (2017).

10. Carrega, P. et al. Nat. Commun. 6, 1-13 (2015).

11. Wang, S. et al. Cell Res. 1-13 https://doi.org/10.1038/s41422-020-0312-y (2020).

12. Moral, J. A. et al. Nature 579, 130-135 (2020). 